\title{
Modeling of RTS Noise in MOSFETs under Steady-State and Large- Signal Excitation
}

J.S. Kolhatkar, E. Hoekstra, C. Salm, A.P. van der Wel, E.A.M. Klumperink, J. Schmitz, H. Wallinga MESA $^{+}$Research Institute, University of Twente, P.O. Box 217, 7500AE, Enschede, The Netherlands. Phone: +31-53-4892727 Fax: +31-53-4891034 Email: j.s.kolhatkar@utwente.nl

\begin{abstract}
The behavior of RTS noise in MOSFETs under large-signal excitation is experimentally studied. Our measurements show a significant transient effect, in line with earlier reports. We present a new physical model to describe this transient behavior and to predict RTS noise in MOSFETs under large signal excitation. With only three model parameters the behavior is well described, contrary to existing models.
\end{abstract}




\section{Modeling of RTS Noise in MOSFETs under Steady-State and Large-Signal Excitation \\ J.S. Kolhatkar, E. Hoekstra, C. Salm, A.P. van der Wel, E.A.M. Klumperink, J. Schmitz, and H. Wallinga \\ $\mathrm{MESA}^{+}$Research Institute, University of Twente, P.O.Box 217, 7500AE, Enschede, The Netherlands. \\ Phone:+31-53-4892727 Fax:+31-53-4891034Email: j.s.kolhatkar@utwente.nl}

\section{Introduction}

With shrinking device dimensions, MOSFETs have become notorious for their Random Telegraph Signal (RTS) noise; limiting the achievable circuit performance in analog and RF CMOS (e.g. LF noise in amplifiers and filters for radio receivers and phase noise due to up-converted LF noise in oscillators).

The steady-state behavior of an RTS has been extensively studied [1-2]. Although it is known that RTS noise changes significantly under largesignal periodic excitation, (Fig.1) [3-5], which is beneficial for oscillators and PLLs, current circuit simulators do not model this behavior [6]. Even circuit simulators that support time-variant noise sources (e.g. periodic steady-state analysis of Spectre RF), do not adequately model the observed effects.

To overcome these shortcomings, we have performed extensive measurements on RTS under large-signal excitation and we present a simple, physical model giving a realistic description of the observed effects.

\section{Experimental}

NMOS transistors with $\mathrm{W} / \mathrm{L}=1 / 0.13$ fabricated in a $0.18 \mu \mathrm{m}$ CMOS process flow are used for this study. The RTS is characterized in the time-domain by three parameters: 1) the mean low time; 2) the mean high time; 3 ) the amplitude (Fig. 2), using a differential measurement setup [5]. Figures 3(a) and 3(b) show the steady-state bias dependence of the mean capture time $\left(\tau_{\mathrm{c}}\right)$, mean emission time $\left(\tau_{\mathrm{e}}\right)$ and RTS amplitude, on the gate-to-source voltage $\left(V_{\mathrm{GS}}\right)$ for a given drain voltage.

In the $O N$ state, the trap-occupancy is given by: $\tau_{\text {e on }} /\left(\tau_{\text {e on }}+\tau_{\text {c on }}\right)$. When the device is in the $O F F$ state for a long period, with no charge carriers in the channel, the trap-occupancy $\left(\tau_{\mathrm{e} \text { off }} /\left(\tau_{\mathrm{e} \text { off }}+\tau_{\mathrm{c} \text { off }}\right)\right)$ is almost zero. After the device is switched $O N$, the trap-occupancy takes time to adapt to this new bias condition. We devise a measurement method to determine this trapoccupancy change from an $O F F$ state to an $O N$ state. To stress the time dependence of the trap-occupancy, we call it instantaneous occupancy (in contrast to the steady-state occupancy). It is determined by averaging the state of the RTS (high or low) over many RTS time-frames, for a given instant during the $O N$ period (Fig. 4). Measurements show that the instantaneous trap-occupancy does not follow the instantaneous stepvoltage but changes exponentially to reach the steady-state value (Fig. 5). Existing circuit simulators implicitly assume an instantaneous change in the trap-occupancy with changing gate-bias, thus giving erroneous noise predictions under periodic bias excitation.

The study of instantaneous trap-occupancy during biasing transients is then extended to the large-signal periodic excitation case. Under periodic largesignal excitation, the device is alternating between an $O N$ state and an $O F F$ state. The excitation frequency is well above the corner frequency of the RTS. Under these conditions the RTS parameters (obtained by sampling the drain-current in the $O N$ states [7]) are significantly different compared to the RTS parameters in the steady-state, resulting in a changed RTS noise spectrum (Fig. 2).

\section{Steady-State RTS Modeling}

Figure 6 shows a block representation of our model. The model is primarily based on the Shockley-Read-Hall statistics of capture and emission [1]. Three physical parameters are used to characterize a trap: the trap energy in silicon band-gap at flat-band $\left(E_{\mathrm{t} 0}\right)$, the location of the trap in the oxide $\left(X_{\mathrm{t}}\right)$, and the intrinsic cross-section of the trap $\left(\sigma_{0}\right)$ [2]. The RTS amplitude is calculated by assuming an elementary electron charge in the channel that changes the channel conductivity [8]. In steady-state, the trap occupancy function follows the Fermi-Dirac statistics and is a function of the trap energy $\left(E_{\mathrm{t}}\right)$, and the Fermi-level for traps $\left(F_{\mathrm{t}}\right)$ [1]. In steady-state, the Fermilevel for the trap $\left(F_{\mathrm{t}}\right)$ is equal to the Fermi-level for the charge carriers in silicon $\left(F_{\mathrm{n}}\right)$ [1], thus, the steady-state trap-occupancy is a function of the band-bending in Silicon for a given $V_{\mathrm{GS}}$. The variation of $\tau_{\mathrm{c}}$ and $\tau_{\mathrm{e}}$ as a function of $V_{\mathrm{GS}}$, is used to extract the trap parameters: $E_{\mathrm{t} 0}, X_{\mathrm{t}}$ and $\sigma_{0}$.

\section{Dynamic RTS Modeling (Periodic large-signal excitation)}

Under periodic large-signal excitation our model assumes $F_{\mathrm{n}}$ changes instantaneously with changes in $V_{\mathrm{GS}}$. During transients, the capture rate is not equal to the emission rate. The RTS behavior under transient biasing conditions shows that the instantaneous trap-occupancy increases exponentially from the $O F F$ state occupancy to the $O N$ state occupancy (Fig. 5). Although the trapping behavior cannot be observed in the $O F F$ state, we expect the $O F F$ transient to be exponential as well. The increase (and decrease) of the trap-occupancy is modeled using $\tau_{\text {OccRlx }}$ (timeconstant for the occupancy relaxation) as a model parameter for the exponential fit. $\tau_{\text {OccRlx }}$ is not a new trap parameter, but the mean time before the trap-occupancy reaches its steady-state value. It can be shown that $\tau_{\text {OccRlx }}$ is the harmonic mean of the $O N(O F F)$ state $\tau_{\mathrm{e}}$ and $\tau_{\mathrm{c}}$, for the $O N$ $(O F F)$ transience [9]. When the frequency of the applied gate-bias is much higher than the corner frequency of the RTS, the average trap-occupancy is somewhere between the steady-state trap occupancy in the $O N$ and $O F F$ state (Fig. 7). Intuitively, this makes sense, as it is clear that slow traps cannot adapt quick enough to fast changes in the biasing, resulting in relaxation effects.

Our model calculates the capture and emission rates from the instantaneous trap-occupancy. For comparing the measurement data with the model results, under periodic large-signal excitation, the averaged $\tau_{\mathrm{c}}$ and $\tau_{\mathrm{e}}$ are calculated using the averaged trap-occupancy and the averaged capture and emission rates (Fig. 6). Figures 8 and 9 show the $\tau_{\mathrm{e}}$ and $\tau_{\mathrm{c}}$ as a function of the duty-cycle and frequency of the large-signal excitation.

Figure 10 shows the variation of $\tau_{\mathrm{e}}$ and $\tau_{\mathrm{c}}$ as a function of the $O F F$ voltage of the applied periodic large-signal. $\tau_{\mathrm{e}}$ decreases rapidly as the $O F F$ voltage goes deeper below the threshold voltage. Physically, this can be visualized as the trap cross-section for emission $\left(\sigma_{\mathrm{e}}\right)$ increasing as the $O F F$ voltage goes deeper below threshold. This behavior is not modeled by assuming a simplistic bias independence of $\sigma_{\mathrm{e}}$. Thus, in our model, the trap cross section decay constant $\left(X_{0}\right)$ [10] is empirically modeled as a function of $V_{\mathrm{GS}}$, to fit our measurement results.

We use our model to simulate the RTS parameters under various biasing conditions (steady-state and periodic large-signal excitation) and match them with measured data by using the same set of extracted trap-parameters (Figures 3, 5, 8, 9, and 10 show measurements and our model simulation on a device RTS1410). The markers in the figures indicate measurement data and solid lines represent our model simulation. Table 1, shows the extracted model trap-parameters for 3 different traps. Finally, figure 11 shows the measurement and simulation of the RTS noise spectrum under steady-state and under large-signal periodic excitation.

\section{Conclusions}

For the first time we investigate trap behavior under transient biasing conditions that is not observable in steady-state, thus providing more insight in trapping and de-trapping mechanisms. We present a simple physics based analytical model to accurately predict the RTS parameters and noise spectra, in steady-state as well as under large signal excitation. All our model parameters have a physical significance and the model shows excellent agreement with measured data on a single RTS. Given a distribution of traps in energy and location, it is possible to extend our model to accurately predict the LF noise in MOSFETs, under varying bias conditions, leading to better optimization of analog and RF designs. 


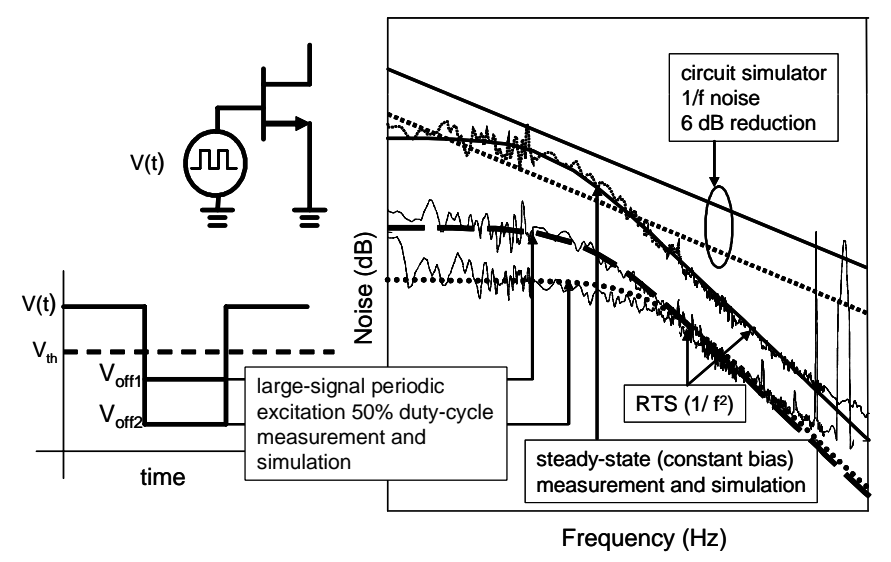

Fig 1: Steady-state LF noise spectrum and LF noise spectra under periodic large-signal excitation for two $O F F$ voltages $\left(V_{\text {off1 }}\right.$ and $V_{\text {off2 }}$ ), measurement and simulated using our model. Also shown is the LF noise predicted by circuit simulator 'Spectre RF' in steadystate and periodic excitation with a $50 \%$ duty-cycle.

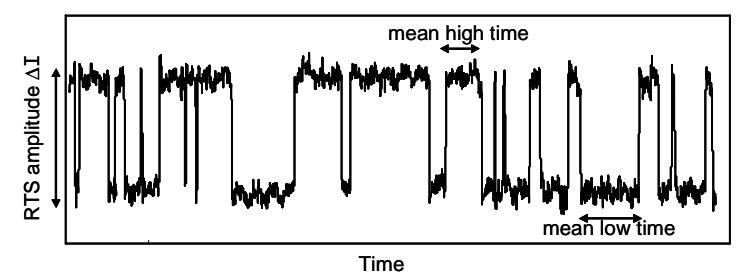

Fig 2: Time-domain trace of the drain current of a MOSFET showing a Random Telegraph Signal (RTS) (parameters: mean high time, mean low time, amplitude).

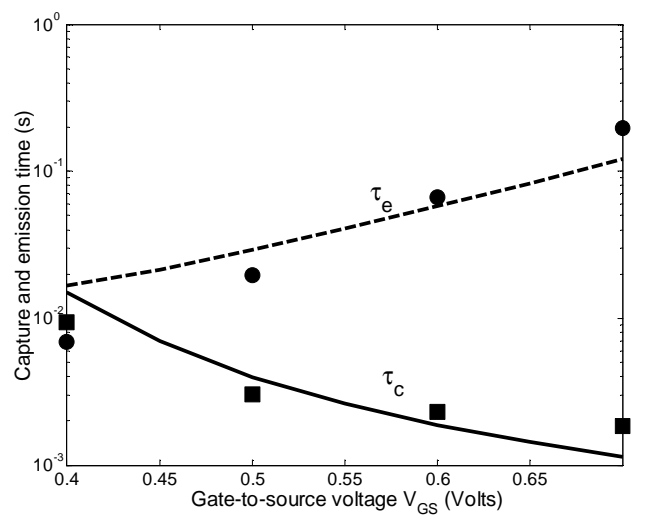

Fig 3(a): RTS time constants $\tau_{\mathrm{c}}$ and $\tau_{\mathrm{e}}$ as a function of $V_{\mathrm{GS}}$ variation for $V_{\mathrm{DS}}=40 \mathrm{mV}$, measurements and simulation.

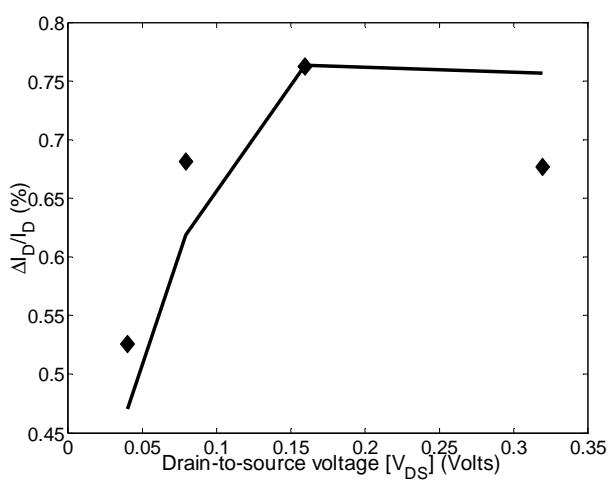

Fig 3(b): RTS amplitude $(\Delta \mathrm{I})$ as a function of $V_{\mathrm{DS}}$ for $V_{\mathrm{GS}}=0.4 \mathrm{~V}$, measurement and simulation.

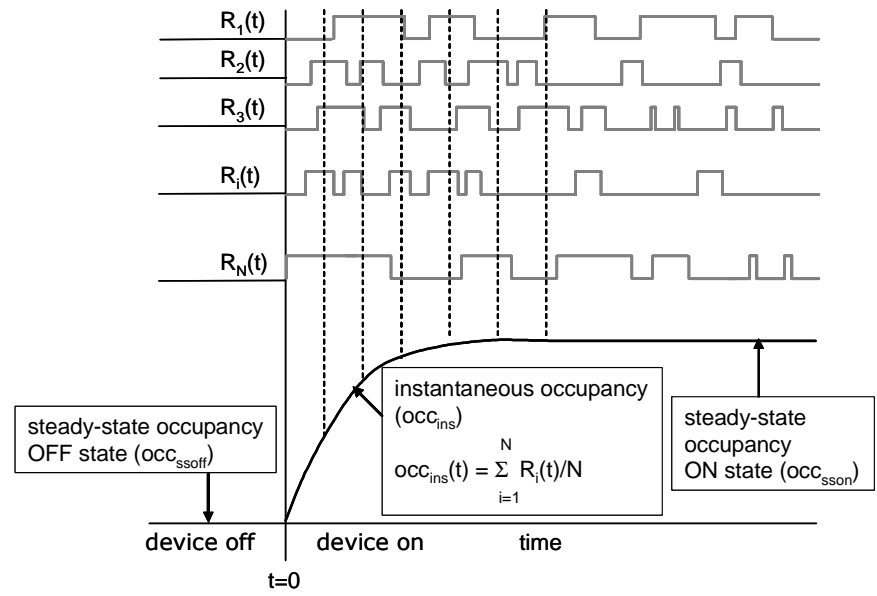

Fig 4: Technique for measuring the instantaneous occupancy. The instantaneous trap occupancy is calculated by averaging the 'state' of the RTS over many RTS frames, during the $O N$ period.

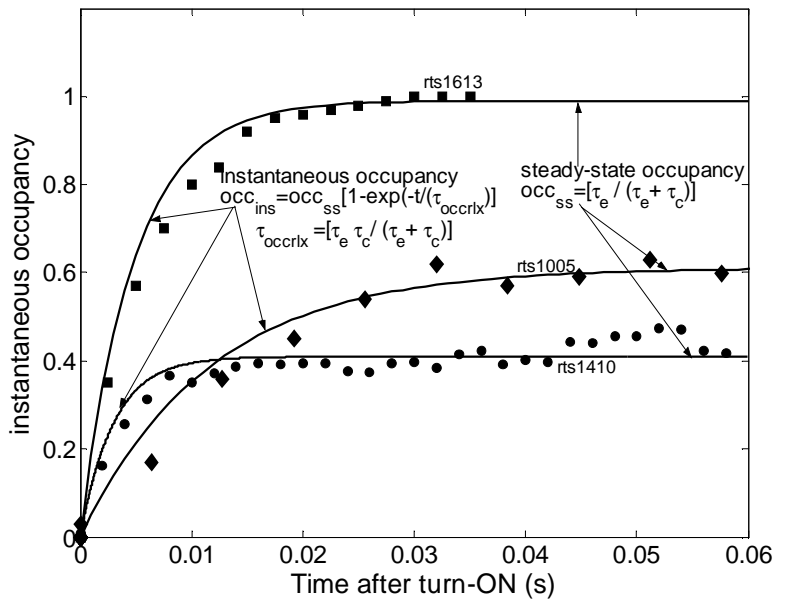

Fig 5: Measured and simulated instantaneous occupancies for 3 different RTS.

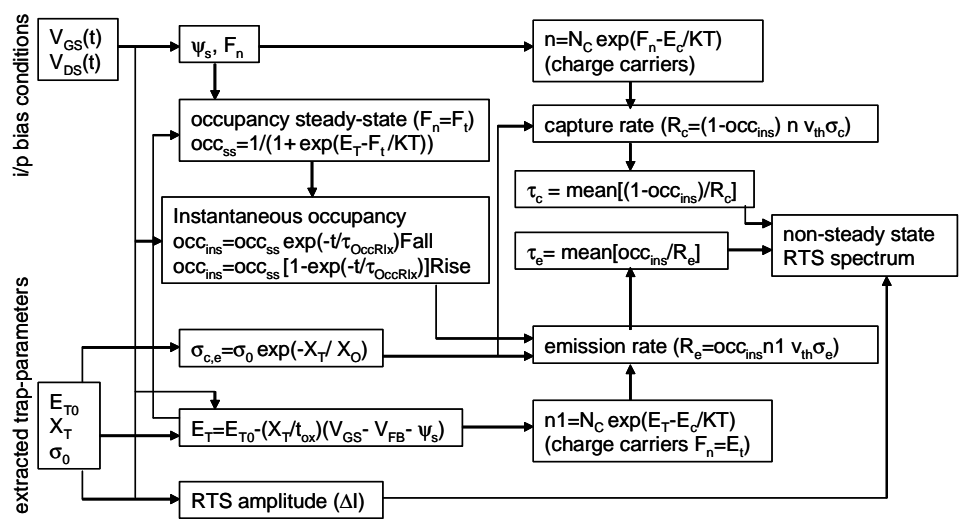

Fig. 6: Block schematic of the proposed model. The model input parameters are the bias conditions and the extracted trap-parameters. The output is the RTS spectra under large-signal excitation. 


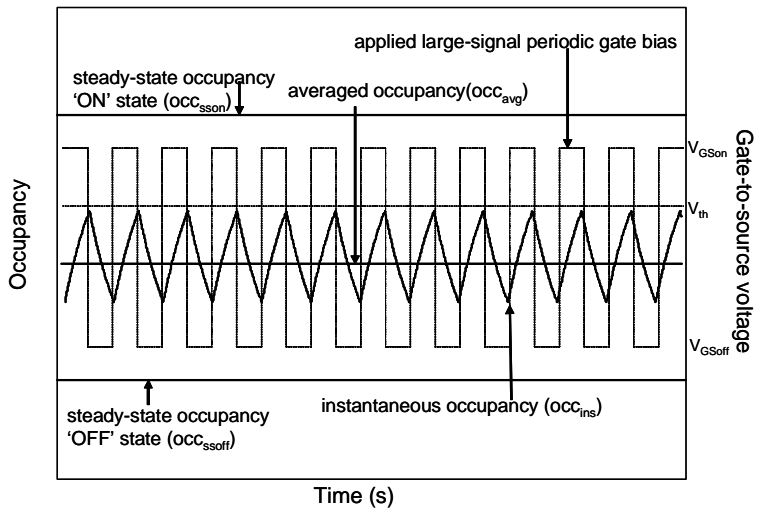

Fig 7: Instantaneous occupancy under periodic large-signal excitation simulated using the model. The excitation frequency $\left(f_{\mathrm{sw}}\right)$ is higher than the corner frequency of RTS. Also shown is the averaged occupancy which lies between the steady-state occupancies in the $O N$ and $O F F$ states.

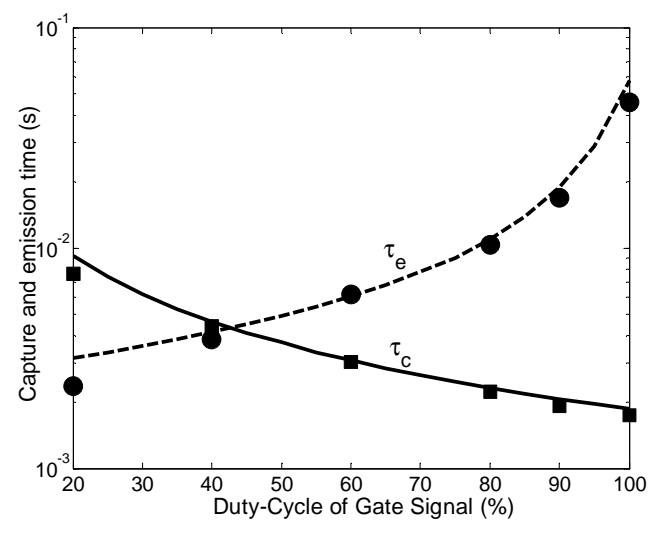

Fig 8: RTS time constants $\tau_{\mathrm{c}}$ and $\tau_{\mathrm{e}}$ as a function of varying dutycycle for $V_{\mathrm{GS} \text { on }}=0.6 \mathrm{~V}, V_{\mathrm{GS} \text { off }}=-0.2 \mathrm{~V}, V_{\mathrm{DS}}=40 \mathrm{mV}, f_{\mathrm{sw}}=10 \mathrm{kHz}$.

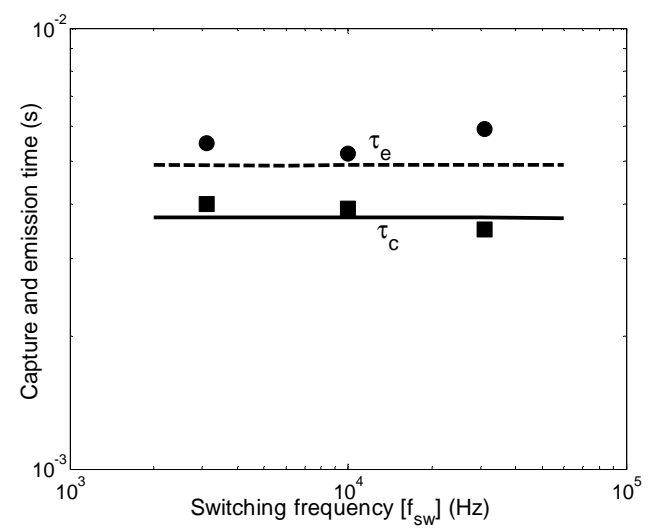

Fig 9: RTS time constants $\tau_{\mathrm{c}}$ and $\tau_{\mathrm{e}}$ as a function of the excitation frequency $\left(f_{\mathrm{sw}}\right)$ for $V_{\mathrm{GS} \text { on }}=0.6 \mathrm{~V}, V_{\mathrm{GS} \text { off }}=-0.2 \mathrm{~V}, V_{\mathrm{DS}}=40 \mathrm{mV}$, duty-cycle $=50 \%$.

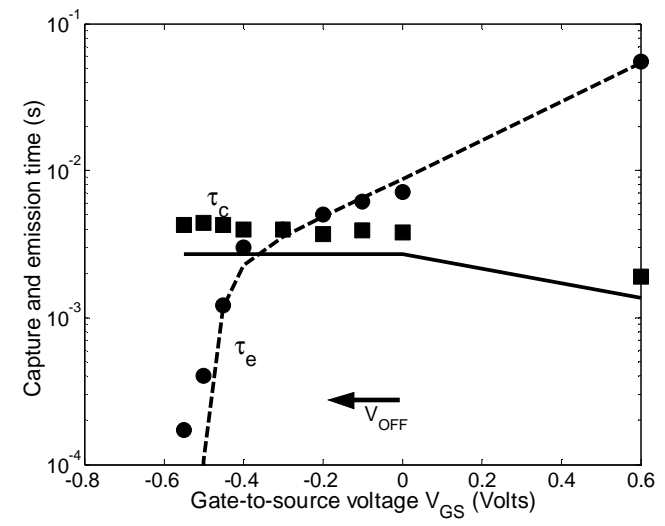

Fig 10: RTS time constants $\tau_{\mathrm{c}}$ and $\tau_{\mathrm{e}}$ as a function of $V_{\mathrm{GS}}$ off for $V_{\mathrm{GS} \text { on }}=0.6 \mathrm{~V}, V_{\mathrm{DS}}=50 \mathrm{mV}, f_{\mathrm{sw}}=31.6 \mathrm{kHz}$.

\begin{tabular}{|l|c|c|c|}
\hline & $E_{\mathrm{t} 0}(\mathrm{eV})$ & $X_{\mathrm{t}}(\mathrm{nm})$ & $\sigma_{0}\left(\mathrm{~cm}^{2}\right)$ \\
\hline RTS1410 & 0.126 & 1.5 & $1 \times 10^{-17}$ \\
\hline RTS1613 & 0.20 & 2.1 & $4 \times 10^{-16}$ \\
\hline RTS0611 & 0.015 & 0.2 & $2.2 \times 10^{-23}$ \\
\hline
\end{tabular}

Table 1: Extracted physical model parameters for different RTS.

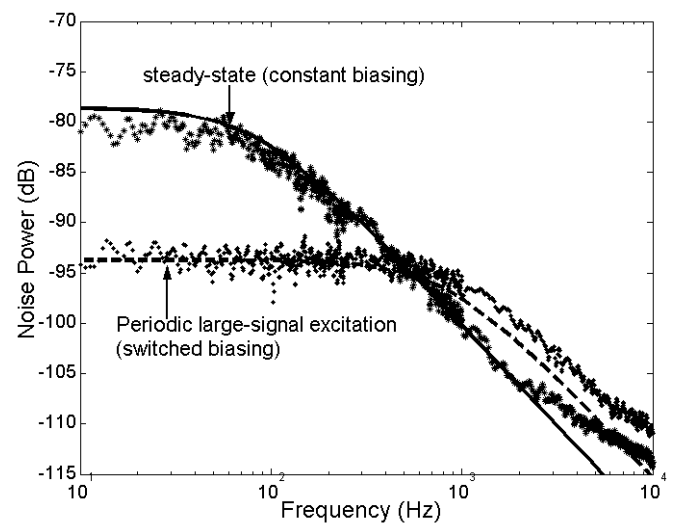

Fig 11: LF noise spectra under steady-state and large-signal periodic excitation. $f_{\mathrm{sw}}=31.6 \mathrm{kHz}, V_{\mathrm{GS}}$ on $=0.6 \mathrm{~V}, V_{\mathrm{GS} \text { off }}=-0.5 \mathrm{~V}$, duty-cycle $=50 \%$.

\section{References:}

[1] W. Shockley et al., Phys. Rev. vol. 871952 pp 835.

[2] M.J. Kirton et al., Adv. Phys. vol 381989 pp 367.

[3] I. Bloom et al., Apl. Phys. Lett. vol. 581991 pp 1664.

[4] B. Dierickx et al., J. Appl. Phys. vol. 711992 pp 2028.

[5] A.P. van der Wel et al., IEEE Elect. Dev. Lett. vol. 212000.

[6] E.A.M. Klumperink et al., IEEE J. Solid-State Cir. vol. 352000 p 994.

[7] J.S. Kolhatkar et al., Proc. ICNF 2003 pp 237.

[8] K.K. Hung et al., IEEE Trans. Elec. Dev. vol. 371990 pp 654.

[9] S. Machlup J. Appl. Phys. vol. 251954 pp 341.

[10] F.P. Heiman et al., IEEE Trans. Elec. Dev. vol. 121965 pp 167. 